Supporting information

\title{
High fluorescent hyperbranched polysiloxane containing $\beta$-cyclodextrin for cell imaging and drug delivery
}

Lihua Bai, ${ }^{\text {a }}$ Hongxia Yan, ${ }^{\text {a }}$ Tian Bai, ${ }^{\mathrm{a}}$ Yuanbo Feng, ${ }^{\mathrm{a}}$ Yan Zhao, ${ }^{\mathrm{a}}$ Yi Ji, ${ }^{\mathrm{a}}$ Weixu Feng, ${ }^{\mathrm{a}}$ Tingli $\mathrm{Lu}^{\mathrm{b}}$, Yufeng $\mathrm{Nie}^{\mathrm{c}}$

a Key Laboratory of Polymer Science and Technology, Shaanxi Province, School of Science, Northwestern Polytechnical University, Xi'an 710129, China.

b Xi'an Key Laboratory of Scientific Computation and Applied Statistics, School of Science, Northwestern Polytechnical University, Xi'an 710129, China.

c Key Laboratory for Space Biosciences \& Biotechnology, Faculty of Life Science, Northwestern Polytechnical University, Xi'an 710072, China.

* Corresponding author. E-mail address: hongxiayan@nwpu.edu.cn

FTIR was used to characterize the synthesized APTES- $\beta-C D$. As shown in Figure S1, the absorption peak at $3358 \mathrm{~cm}^{-1}$ of $\beta-\mathrm{CD}$ belongs to the stretching vibration of $-\mathrm{OH}$. Peaks at 2931,1453 and $1034 \mathrm{~cm}^{-1}$ are attributed to the stretching vibration of $-\mathrm{CH}_{2-}$, deformation 
vibration of $-\mathrm{CH}_{2}$ - and stretching vibration of C-O-C, respectively. In the FTIR spectrum of APTES, absorption peaks at $3441,1647,1108$ and $780 \mathrm{~cm}^{-1}$ are ascribed to the stretching vibration of $-\mathrm{NH}_{2}$, bending vibration of $-\mathrm{NH}_{2}$, stretching vibration of $\mathrm{Si}-\mathrm{O}$ and $\mathrm{Si}-\mathrm{C}$, separately. In the FTIR of APTES- $\beta-C D$, the absorption peaks of $\beta-C D$ and APTES are presented, especially the stretching vibration of Si-O and C-O-C at 1108 and $1034 \mathrm{~cm}^{-1}$. Thus, APTES- $\beta$ CD has been successfully synthesized.

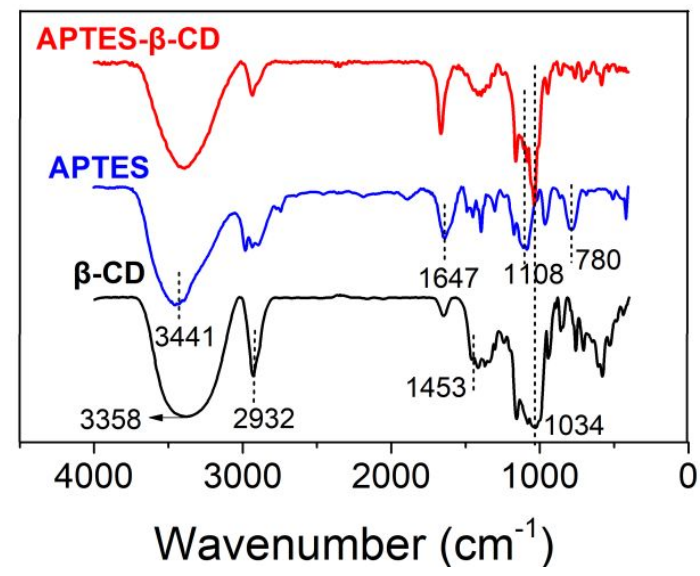

Figure S1. FTIR of $\beta$-CD, APTES and CD-APTES.

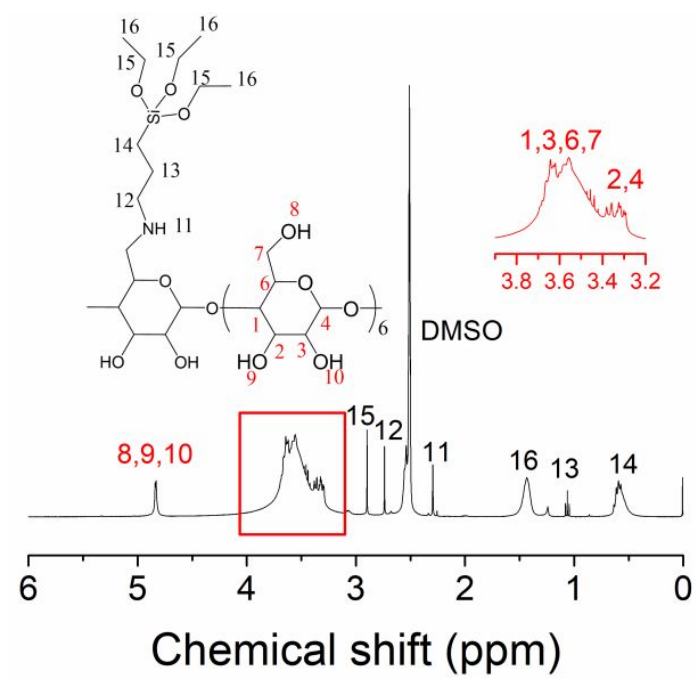

Figure S2. ${ }^{1} \mathrm{H}$ NMR spectra of APTES- $\beta-C D$. 

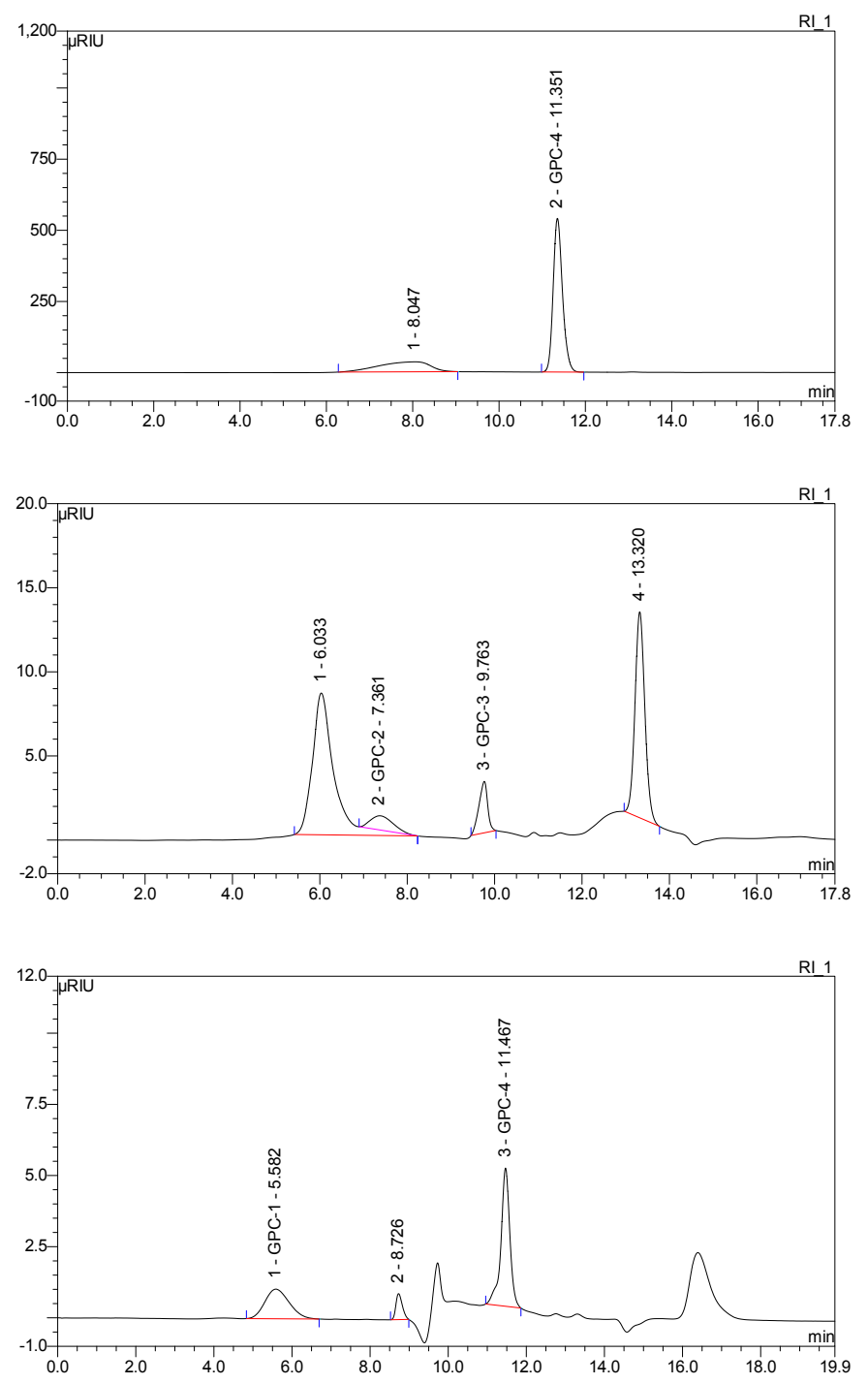

Figure S3. GPC curves of HBPSi, HBPSi-CD1 and HBPSi-CD2 (from top to down).

Hyperbranched polysiloxanes is a novel kind of nonconjugated fluorescent polymer. However, the weak fluorescence intensity limits its application. To improve the fluorescence intensity of HBPSi, $\beta$-CD (rigid molecule with plenty of hydroxyls) was introduced to improve the rigidity and aggregation of HBPSi. Hyperbranched polysiloxanes was synthesized via one pot melting polycondensation of APTES and NMDEA. Then two light yellow solid HBPSiCDs, with different molecular weights, were synthesized by varying the mole ratio of HBPSi and CD-APTES, as shown in Table 1 . The relative content of $\beta$-CD was calculated from ${ }^{13} \mathrm{C}$ 
NMR spectra (Figure S4). It is found that the relative contents of $\beta-C D$ are 33 and $43 \%$, named HBPSi-CD1 and HBPSi-CD2, respectively.

Table S1 Reaction Conditions and characterization data of the resultant polymers

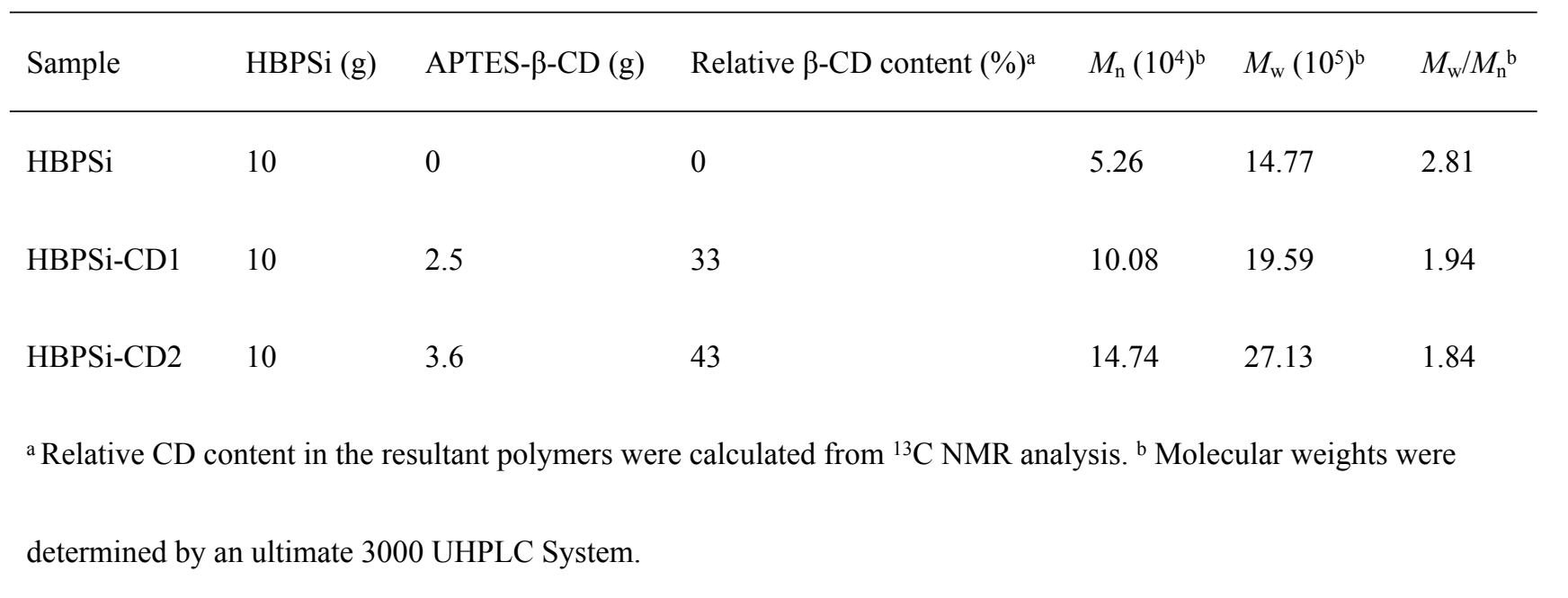
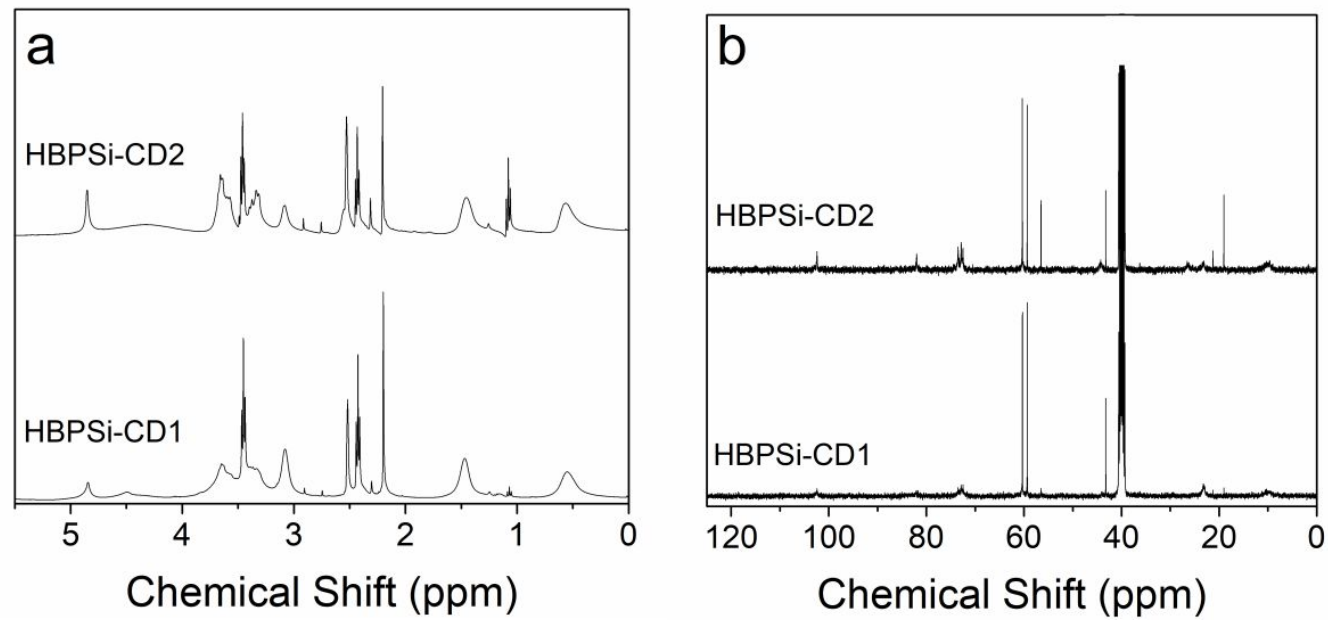

Figure S4. ${ }^{1} \mathrm{H}$ (a) and ${ }^{13} \mathrm{C}(\mathrm{b}) \mathrm{NMR}$ spectra of HBPSi-CD1 and HBPSi-CD2.

NMR and FTIR analysis (Figure S5 and S6) show that, chemical bond formation (amido bond) and encapsulation are the main way of the drug loading. At $\mathrm{pH} 6.4$, the chemical bond between ibuprofen and HBPSi-CD is much easier to be hydrolyzed, meanwhile HBPSi-CD and ibuprofen are all positively charged, the electrostatic repulsion effectively promotes the drug release. 


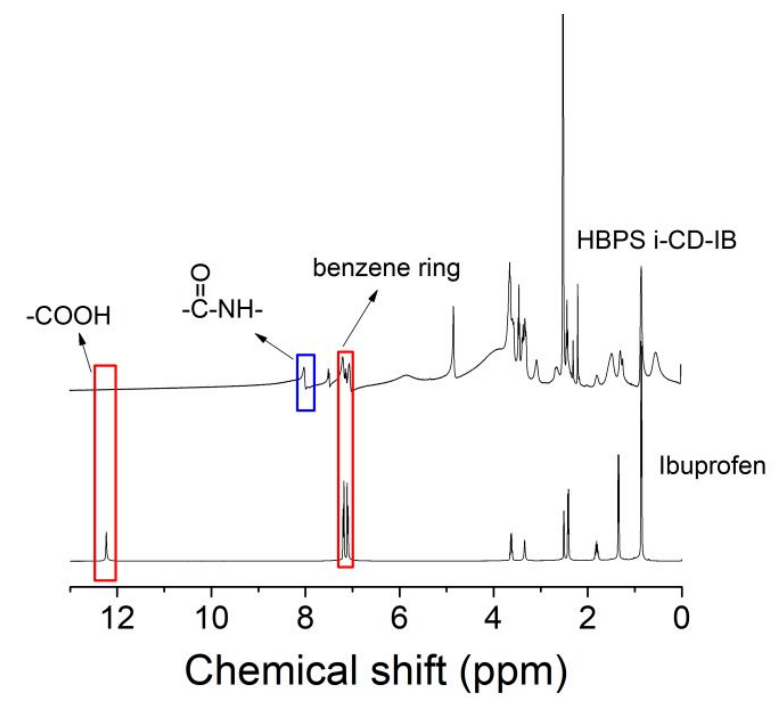

Figure S5. ${ }^{1} \mathrm{H}$ NMR spectra of HBPSi-CD2 before and after ibuprofen is loaded.

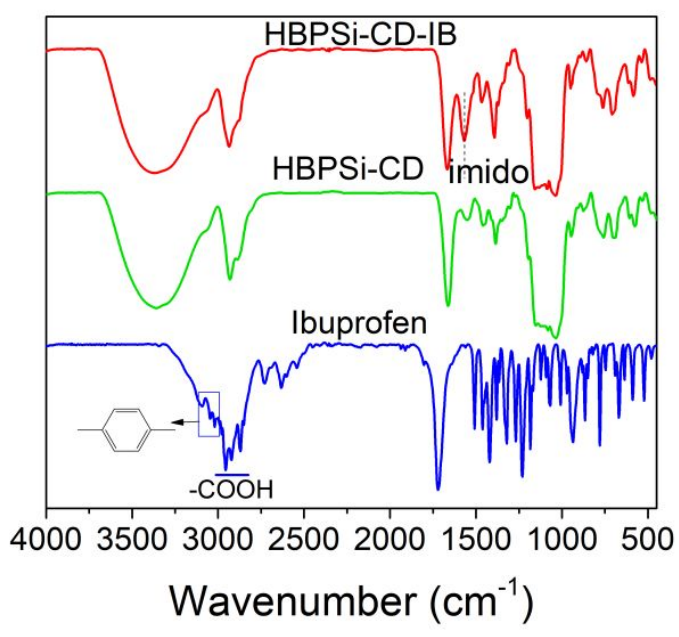

Figure S6. FTIR of HBPSi-CD2 before and after ibuprofen is loaded.

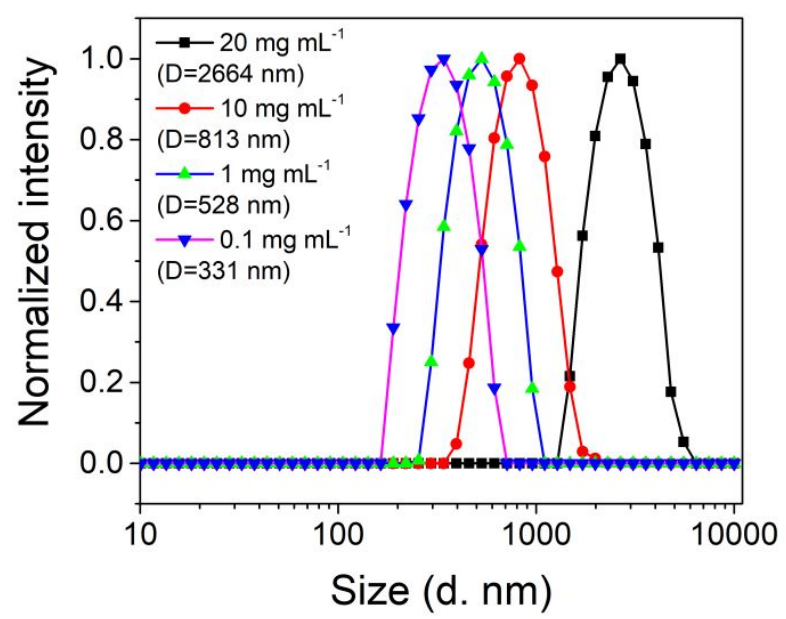

Figure S7. Size distribution of HBPSi-CD2 in aqueous solution with different 
concentrations.

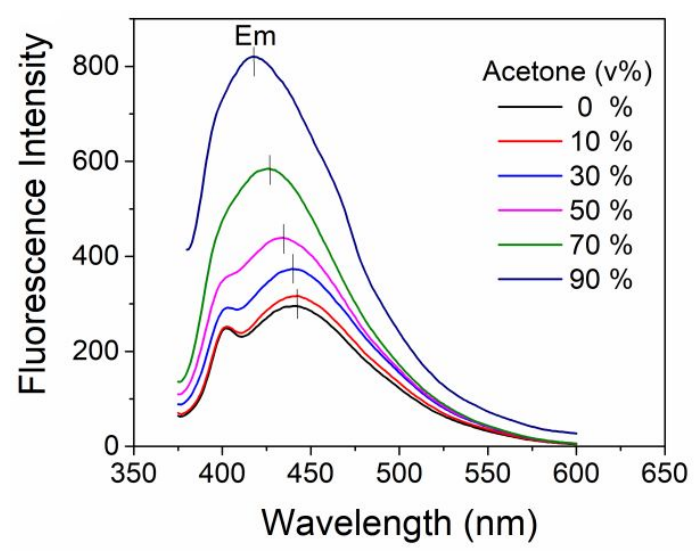

Figure S8. Emission spectra of HBPSi-CD1 in water-acetone mixture $\left(0.1 \mathrm{mg} \mathrm{mL}^{-1} ; \mathrm{Ex}=350\right.$ $\mathrm{nm})$.
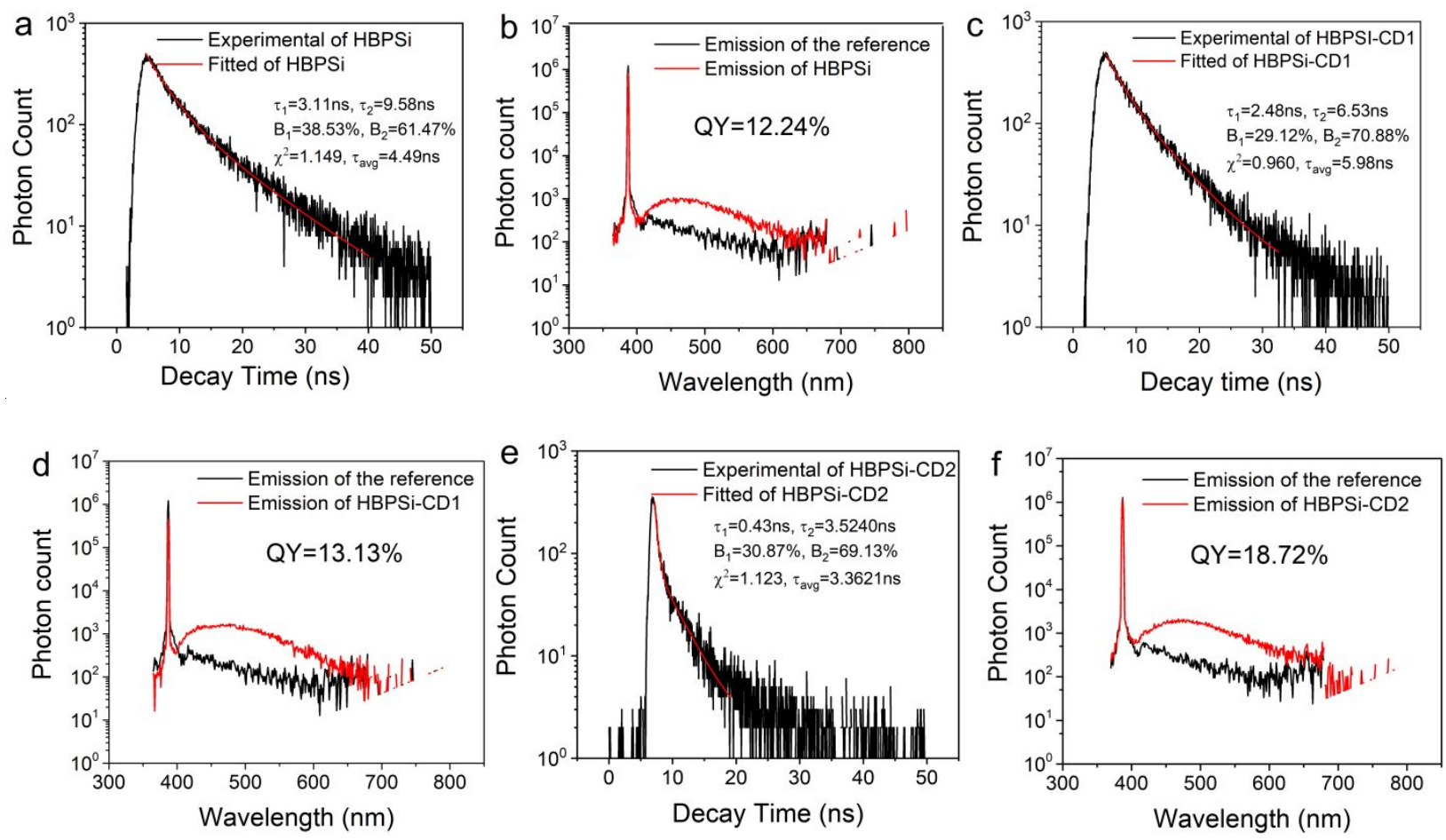

Figure S9. The transient photoluminescence decay curve of HBPSi at $450 \mathrm{~nm}$ after excitation at $387 \mathrm{~nm}$ (a), HBPSi-CD1 at $453 \mathrm{~nm}$ after excitation at $387 \mathrm{~nm}$ (c) and HBPSi-CD2 at 454 $\mathrm{nm}$ after excitation at $387 \mathrm{~nm}(\mathrm{e})$; the absolute fluorescence quantum yield (QY) of HBPSi excited at $387 \mathrm{~nm}$ (b), HBPSi-CD1 excited at $387 \mathrm{~nm}$ (d) and HBPSi-CD2 at $387 \mathrm{~nm}$ (f). 
were measured. As shown in Figure S9, the transient photoluminescence decay curve of HBPSi is located at $450 \mathrm{~nm}$ after excitation at $387 \mathrm{~nm}$. The decay curve was then fitted on the nonlinear least-squares analysis via the following equation: $R(t)=B_{1} \exp \left(-t / \tau_{1}\right)+B_{2} \exp \left(-t / \tau_{2}\right)+B_{3}$ $\exp \left(-t / \tau_{3}\right)$, where $B_{1}, B_{2}$ and $B_{3}$ are the fractional contributions of the time-resolved decay lifetime of $\tau_{1}, \tau_{2}$ and $\tau_{3}$, respectively. Then, the average fluorescence lifetimes $\left(\tau_{\text {avg }}\right)$ of the polymers were calculated by $\tau_{\text {avg }}=\left(B_{1} \tau_{1}^{2}+B_{2} \tau_{2}^{2}+B_{3} \tau_{2}^{3}\right) /\left(B_{1} \tau_{1}+B_{2} \tau_{2}+B_{3} \tau_{3}\right)$. The decay curves of the three polymers fit the double exponential function, and the $\tau_{\text {avg }}$ of HBPSi, HBPSi-CD1 and HBPSi-CD2 are 8.49, 5.99 and $3.36 \mathrm{~ns}$, respectively. The absolute quantum yields of HBPSi, HBPSi-CD1 and HBPSi-CD2 reach to $12.24,13.13$ and $18.72 \%$.

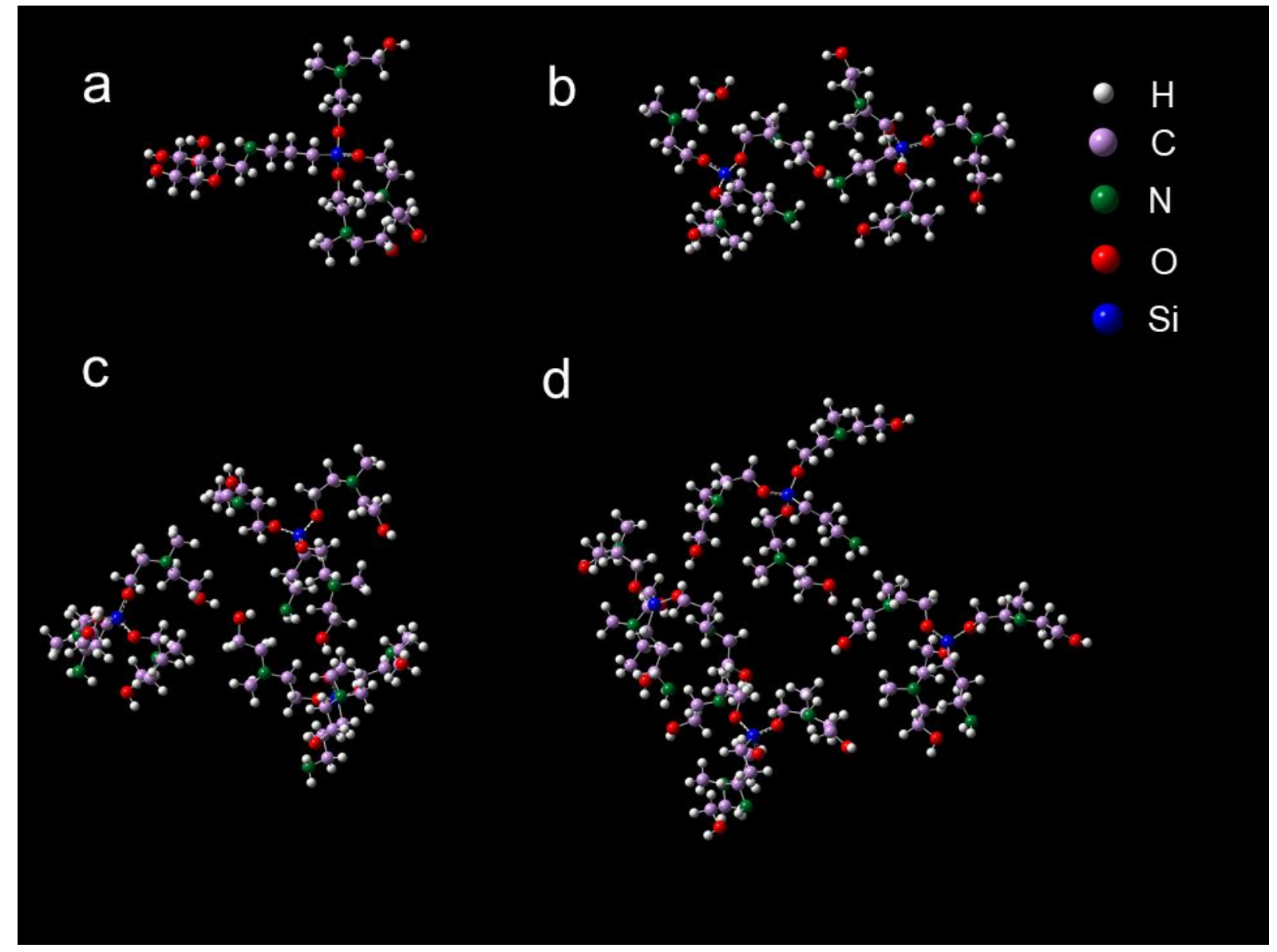

Figure S10. Optimized conformations of various HBPSi molecules: one molecule (a), two molecules (b), three molecules (c) and four molecules (d). 


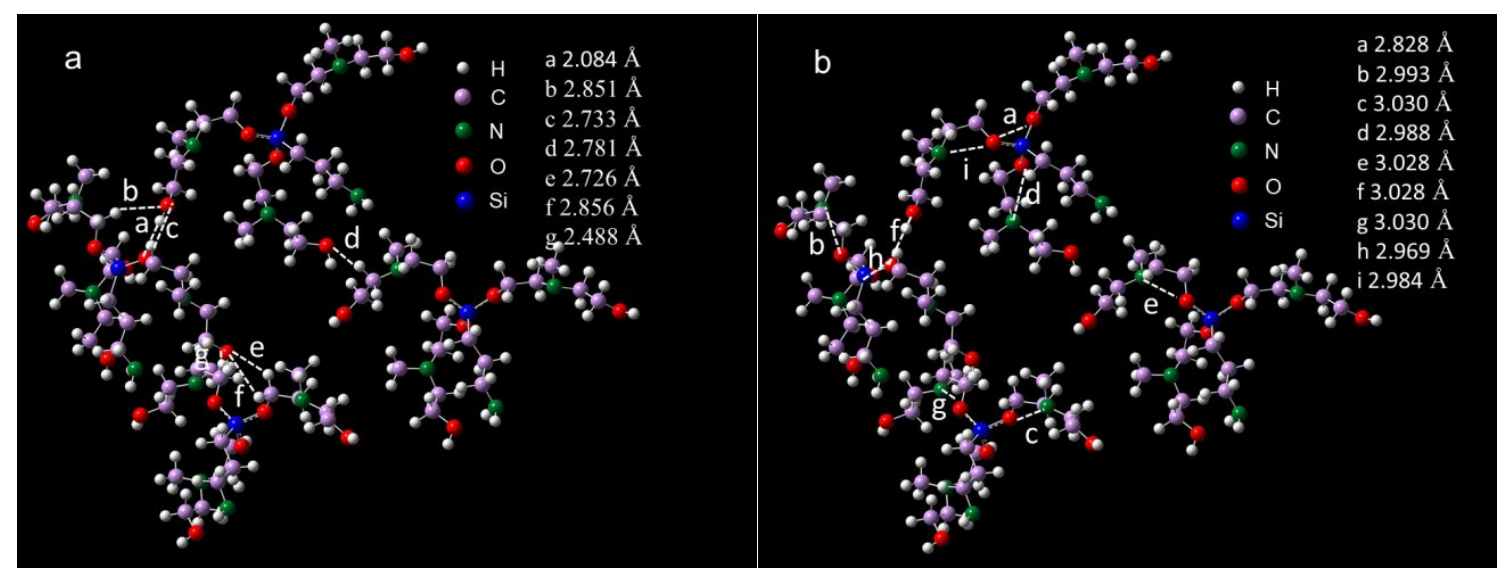

Figure S11. Hydrogen bonds between four HBPSi molecules (a). Intramolecular and intermolecular $\mathrm{O} \cdots \mathrm{O}$ and $\mathrm{O} \cdots \mathrm{N}$ interactions in four HBPSi molecules (b).

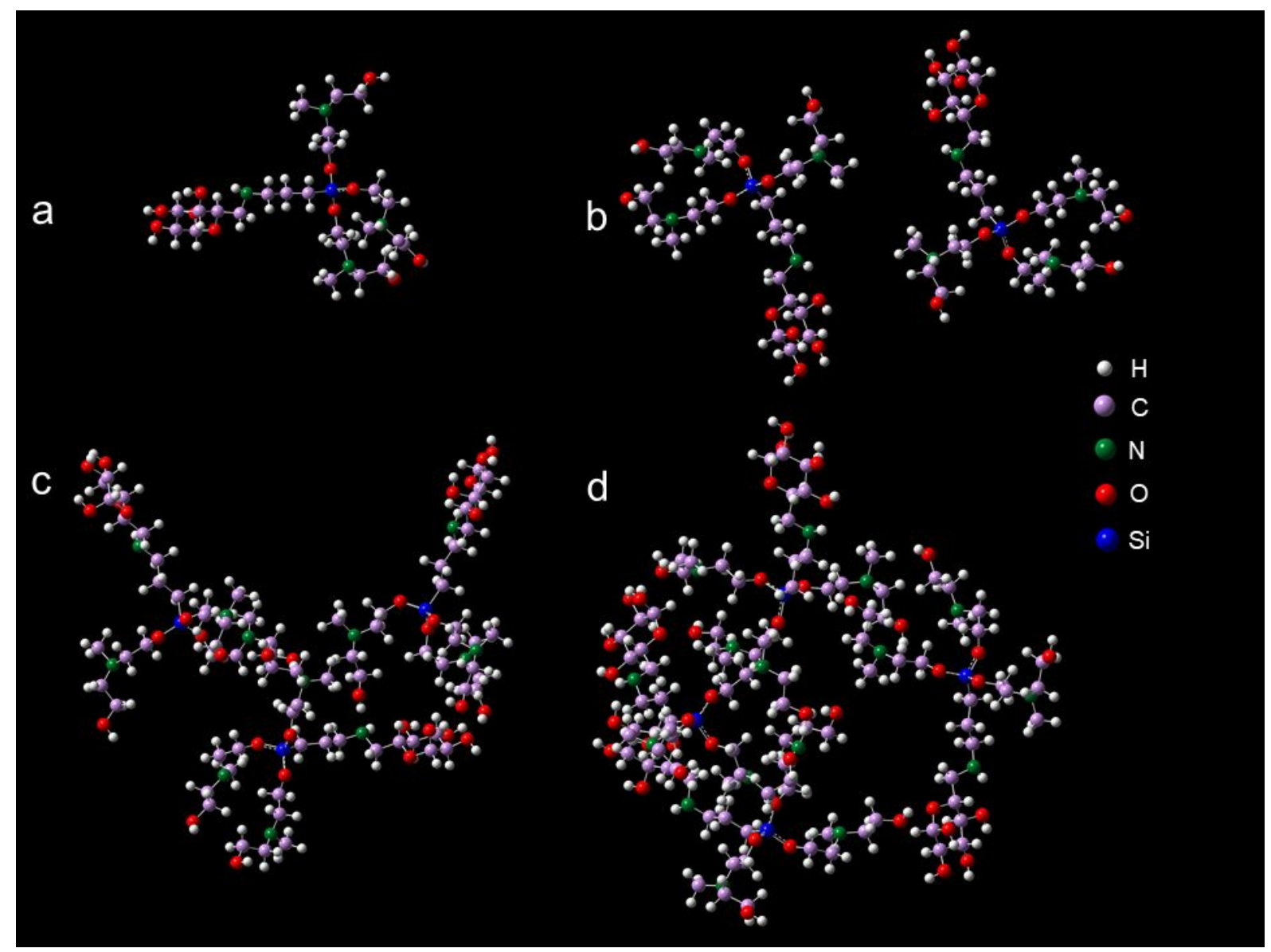

Figure S12. Optimized conformations of various HBPSi-CD molecules (a) One molecule.(b)

Two molecules.(c)Three molecules.(d)Four molecules. 


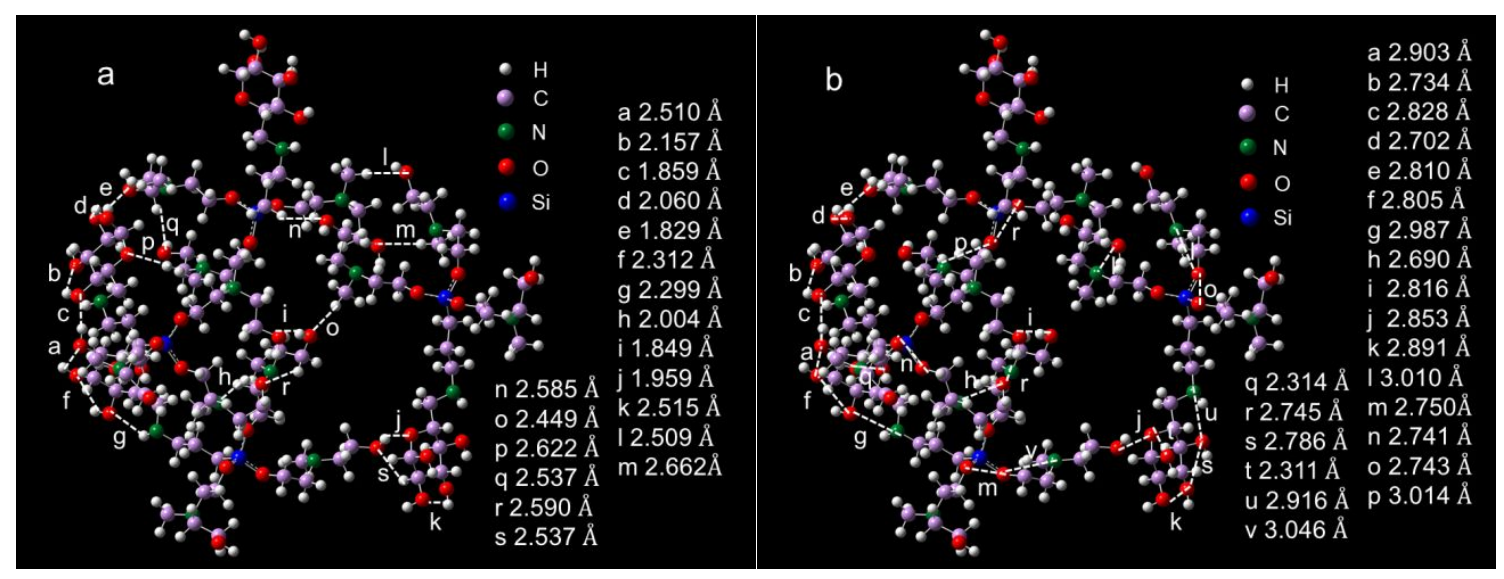

Figure S13. Hydrogen bonds between four HBPSi-CD molecules (a). Intramolecular and intermolecular $\mathrm{O} \cdots \mathrm{O}$ and $\mathrm{O} \cdots \mathrm{N}$ interactions in four HBPSi molecules (b).
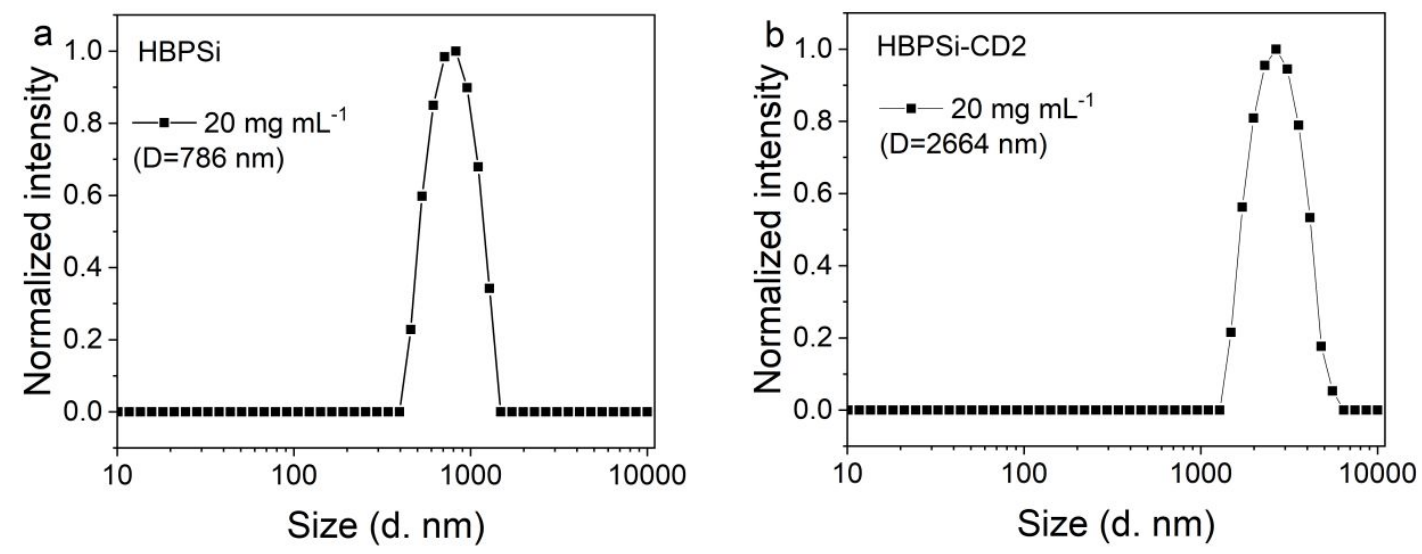

Figure S14. Size distribution of the self-assemblies of HBPSi and HBPSi-CD2 in water solution $\left(20 \mathrm{mg} \mathrm{mL}^{-1}\right)$. 
Table S2. DFT calculation results of HOMO-LUMO energy levels of the conformations with various molecules

\begin{tabular}{cccccc}
\hline \multirow{2}{*}{ sample } & Molecule number & E(HOMO)/a.u. & E(LUMO)/a.u. & Energy gap/a.u. & Energy gap/eV \\
\hline \multirow{3}{*}{ HBPSi } & 1 & -0.210 & 0.058 & 0.268 & 7.287 \\
& 2 & -0.210 & 0.056 & 0.266 & 7.230 \\
& 3 & -0.201 & 0.046 & 0.247 & 6.720 \\
& 4 & -0.180 & 0.058 & 0.238 & 6.473 \\
\multirow{3}{*}{ HBPSi-CD } & 1 & -0.206 & 0.035 & 0.241 & 6.559 \\
& 2 & -0.206 & 0.036 & 0.242 & 6.580 \\
& 3 & -0.189 & 0.035 & 0.224 & 6.093 \\
\hline
\end{tabular}

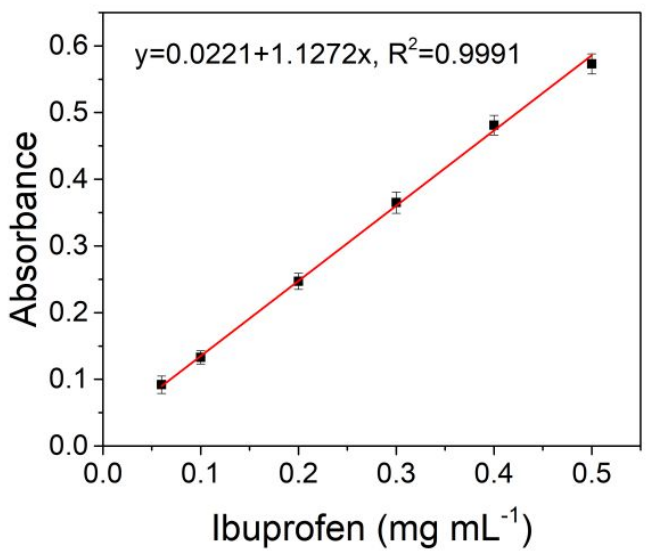

Figure S15. UV-vis absorbance standard curve of ibuprofen in ethanol at $265 \mathrm{~nm}$.

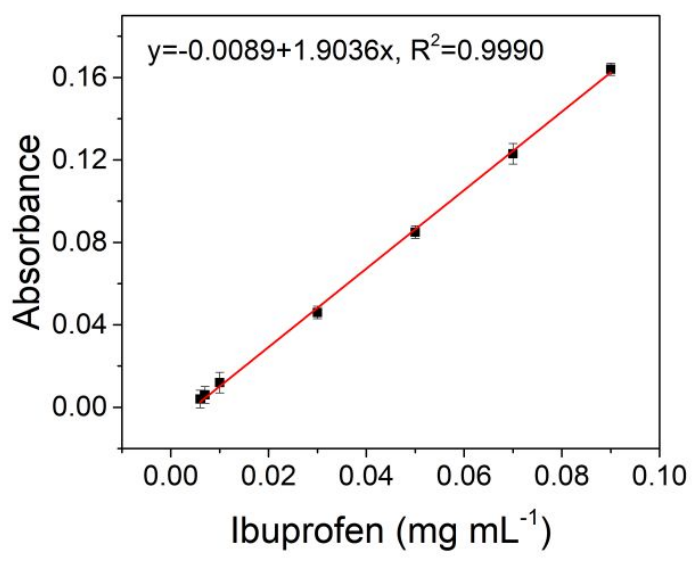

Figure S16. UV-vis absorbance standard curve of ibuprofen in $\mathrm{pH}=6.4 \mathrm{PBS}$ solution at 265 nm. 


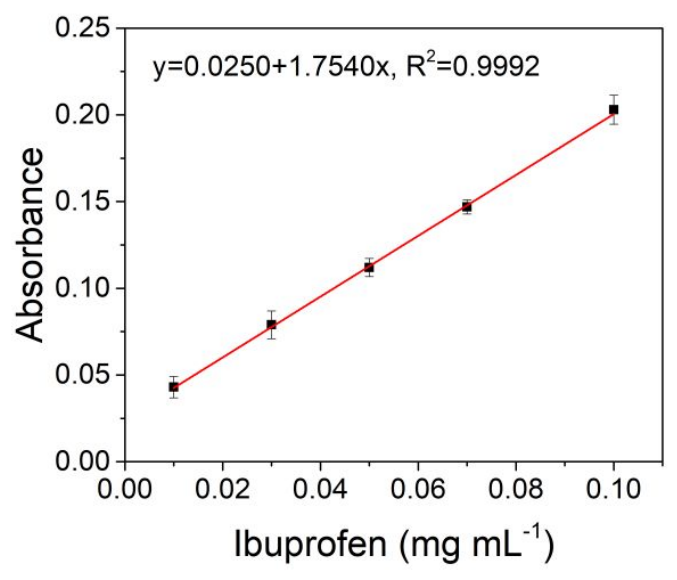

Figure S17. UV-vis absorbance standard curve of ibuprofen in $\mathrm{pH}=7.4 \mathrm{PBS}$ solution at 265 nm.

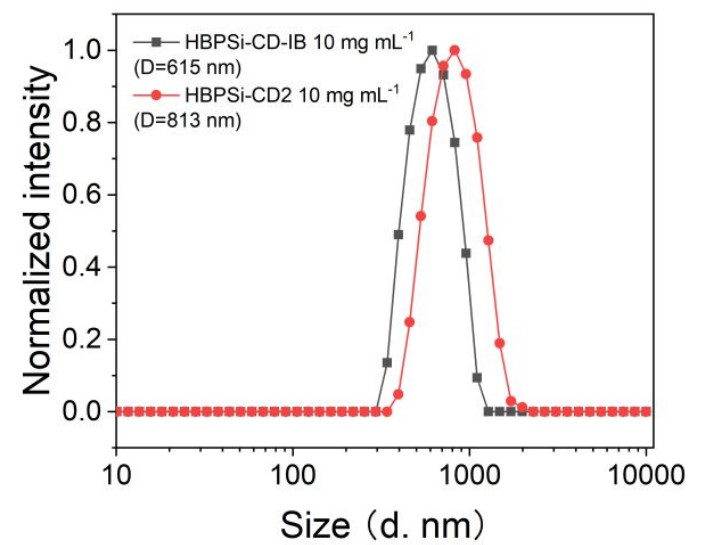

Figure S18. Size distribution of the self-assemblies of HBPSi-CD2 in water solution before and after loading ibuprofen $\left(10 \mathrm{mg} \mathrm{mL}^{-1}\right)$. 